\title{
Evaluación de recursos audiovisuales para la enseñanza de las Ciencias Sociales en Educación Secundaria
}

\author{
Evaluation of Audiovisual Materials for the Teaching of Social \\ Science in Secondary Education
}

\author{
Laura Arias-Ferrer \\ Alejandro Egea-Vivancos \\ Fuensanta Monroy-Hernández \\ Universidad de Murcia
}

Recibido: $13 / 03 / 2019$

Aceptado: 27/06/2019

\begin{abstract}
The broad literature on the use of audiovisual aids in teaching has paid little attention to how specific resources may promote learning of social sciences among secondary students. This study analyzed the extent to which a number of audiovisual resources used in documentaries contributed to student understanding of specific contents. A sample of 120 secondary education students (44,1\% males, $55,9 \%$ females; mean age: 15.7$)$ from three high schools in southeast Spain watched a 15-minute excerpt of three documentaries on historical or geographical topics, and completed an ad hoc questionnaire with open-ended comprehension questions. The results showed that students best recalled facts introduced by a presenter or reader, o by means of visual resources such as maps, photographs, timelines or graphs, which support findings of similar studies on retention through video-based multimedia instruction. Nevertheless, an overload of information presented in a video in a short period of time may prove counterproductive and have a negative effect on understanding and learning, thus causing the so-called redundancy effect. Future research is needed and should focus on examining the impact of specific audiovisual resources (combined or in isolation) used in history and/or geography documentaries on students' ability to retain and recall contents.
\end{abstract}

KEY WORDS: resource evaluation, Social Sciences, educational documentaries, quatitative techniques, audiovisual teaching.

\begin{abstract}
RESUMEN
Las posibilidades educativas de los audiovisuales son hoy día incuestionables, si bien su uso, utilidad y cómo influyen determinados recursos visuales en la enseñanza de las Ciencias Sociales, concretamente de la Historia y la Geografía, cuenta aún con escasos estudios. La presente investigación de corte evaluativo analizó los recursos empleados en tres audiovisuales que diferían entre sí en temática, enfoque y estilo. Una muestra de 120 estudiantes de Educación Secundaria (edad media: 15.7 años) seleccionados por conveniencia visionó un fragmento de aproximadamente 15 minutos de cada uno de los tres audiovisuales, todos ellos de tipo documental y contenido histórico y patrimonial. El objetivo fue identificar qué recursos ayudaban más a los estudiantes a entender determinados contenidos y, por lo tanto, favorecían el recuerdo de los mismos. Tras cada visualización los participantes cumplimentaron un instrumento compuesto de preguntas abiertas que medían la comprensión y recuerdo de aspectos históricos o geográficos y de contenidos conceptuales asociados al uso de determinadas estrategias. Los resultados mostraron que los participantes recordaban mejor aquellos elementos enunciados por un locutor/presentador pero acompañados de soporte gráfico, si bien un exceso de información presentada en un breve espacio de tiempo podía ser contraproducente para el aprendizaje (redundacy effect). Este estudio concluye pues la importancia de introducir la información, previamente seleccionada, por diversas vías, siendo los apoyos gráficos el mejor acompañamiento al discurso oral emitido.
\end{abstract}

PALABRAS CLAVE: evaluación de medios, Ciencias Sociales, documentales educativos, técnicas cuantitativas, didáctica audiovisual.

\footnotetext{
Dirección de correspondencia:

Alejandro Egea-Vivancos. Departamento de Didáctica de las Ciencias Matemáticas y Sociales. Facultad de Educación. Universidad de Murcia (España). E-mail: alexegea@um.es. ORCID: https://orcid.org/0000-0002-6047-2670

Laura Arias-Ferrer. Departamento de Didáctica de las Ciencias Matemáticas y Sociales. Facultad de Educación. Universidad de Murcia (España). E-mail: larias@um.es. ORCID: https://orcid.org/00000003-3121-1882

Fuensanta Monroy-Hernández. Departamento de Métodos de Investigación y Diagnóstico en Educación. Facultad de Educación. Universidad de Murcia (España). E-mail: fuensanta.monroy@um.es. ORCID: https://orcid.org/0000-0002-9813-3068
} 


\section{Introducción}

A pesar de que en pleno siglo XXI las posibilidades educativas de lo audiovisual (ya sea documental, cine, televisión o multimedia) parecen estar fuera de dudas e incluso superadas y que, en general, los medios audiovisuales son muy utilizados en la enseñanza desde hace ya muchas décadas (Dale, 1969; Fairgrieve, 1932), la realidad es que la investigación empírica sobre las posibilidades educativas de muchos de estos recursos aún está en construcción (Mayer, 2009; Mitra, Lewin-Jones, Barrett \& Williamson, 2010). Al respecto, es paradigmático el caso de la serie de estudios realizados sobre el programa infantil «Barrio Sésamo» (Anderson, 1998; Ball \& Bogatz, 1970; Cole \& Lee, 2016; Connell \& Palmer, 1971; Fisch, 2004). Posiblemente se esté ante uno de los vídeos didácticos mejor diseñados, y es que no hay que olvidar que el diseño es clave en el poder educativo de estos productos. En este sentido, cabe detenerse en diferenciar cierta terminología que se va a emplear, en concreto en la diferencia entre un vídeo didáctico y un vídeo educativo (Salinas, 1992), donde el primero está expresamente diseñado para la enseñanza mientras que el segundo puede acabar siéndolo cualquiera, en base a cómo se utilice.

Entre los segundos se encuentra el cine, que ha sido empleado por muchos docentes para intentar conseguir una mayor motivación en sus clases. Por esa razón, las investigaciones sobre el poder didáctico del cine sí que son algo más numerosas. El cine se muestra como un gran recurso para favorecer lo que algunos denominan «aprendizaje integrado», ya que permite conectar y relacionar entre sí contenidos propios de diversas disciplinas, exigiendo al alumnado la movilización de todos sus conocimientos (historia, geografía, política, ética, ciencia, tecnología, etc.) (Torre, 2005). Además, es bastante frecuente que su inclusión en la educación formal y no formal vaya acompañado de materiales didácticos creados ex profeso (Moya, 2017).

En la enseñanza de la historia, son habituales las referencias al cine histórico, ya sea como elemento motivador o como potenciador de la capacidad crítica del estudiante (Ballesteros, 2016; Del Alcàzar, 2012; Donnelly, 2014; Martínez, 2013). El cine histórico se configura en general como un arma dinámica educativa fundamental para trabajar y desmontar clichés, ideas erróneas, estereotipos culturales asumidos de manera acrítica por la población en general y por el público juvenil en concreto. También son frecuentes las recopilaciones de títulos de películas, series y documentales que sirven para trabajar esta disciplina en el aula (Breu, 2012), así como propuestas concretas de adaptación al aula (Autor, 2013a, 2013b; Carrasco, 2017; Grupo «Imágenes de la Historia», Gorgues \& Goberna, 1998; Valero \& Vera, 2005; Valle, 2007). Estos recursos permiten incluso "meterse en el mundo virtual de los personajes y vivir con ellos lo que están viviendo" (Torre, 2005, p. 18), lo que convierte al recurso audiovisual y cinematográfico en uno altamente recomendado por numerosos didactas de la historia (Breu, 2012; Del Alcàzar, 2012; Fernández, 1988; Grupo «Imágenes de la Historia» et al., 1998).

En el caso de la geografía sucede algo similar. Desde las primeras reflexiones sobre la necesidad de incluir películas para su enseñanza (Fairgrieve, 1932, 1942), son habituales las adaptaciones de ciertas películas o documentales para trabajar elementos físicos o humanos de la geografía (Aitken,1994; Algeo, 2007; Hollingham, 1997; Palma, 2009) o incluso reflexiones sobre las posibilidades de ciertos programas de televisión (Durbin, 1995). También se ha recomendado su uso para trabajar de una manera activa la ciudadanía, reforzando ciertos valores a través de lo audiovisual (Castro, 2006; Zaplana, 2003).

Todos estos estudios parten de la base de que el recurso audiovisual, por sí mismo, permite captar la atención del alumnado y mostrar de manera gráfica y visual los contenidos curriculares a impartir en el aula. Pero, en realidad, el vídeo per se no enseña (Bravo, 1996; Laurillard, 2002). En este sentido, y salvo excepciones (Ijaz, Bogdanovych, \& Trescak, 2017), es prácticamente yermo el campo sobre investigaciones de tipo evaluativo sobre vídeos de Ciencias Sociales en general, o de Historia o Geografía en particular, que justifique estas acciones. De aquí el carácter novedoso de esta investigación. 
Se considera fundamental analizar hasta qué punto los recursos audiovisuales promueven el aprendizaje de los contenidos que muestran. La investigación de corte evaluativo permite dar respuesta a este objetivo, así como analizar el grado de adecuación y pertinencia de los audiovisuales al nivel educativo para el que hayan sido diseñados y según los objetivos para los que hayan sido concebidos. Determinar qué es eficaz educativamente hablando es complejo e incluye variables como coste, comprensión, recuerdo, motivación, etc. (Salinas, 1992). Desgraciadamente, las investigaciones evaluativas de un determinado audiovisual que analicen los recursos que favorecen la motivación, aprendizaje o retención de conceptos, o que comparen varios vídeos o diversos formatos, son todavía escasas (Gambari, Shittu, Daramola \& James, 2016; Gambari, Yaki, Gana \& Ughovwa, 2014; Ijaz, Bogdanovych, \& Trescak, 2017), de ahí la necesidad de llevar a cabo investigaciones como la que aquí se presenta.

De la importancia de la incorporación y uso de determinados recursos para el diseño de vídeos didácticos, concretamente aquellos en forma de documentales, versa esta investigación. Para ello, se parte de diferentes audiovisuales de tipo documental desarrollado por la Fundación Integra. Desde 1998, dicha institución ha focalizado una gran parte de su actividad en la generación de múltiples recursos digitales, centrados especialmente en favorecer la difusión del patrimonio de la Región de Murcia entre los más jóvenes. De todos los producidos hasta el momento, una mayoría poseen un contenido histórico y patrimonial. Si bien, los modelos, enfoques o recursos escogidos han sido variados. Ninguno se había diseñado como un vídeo didáctico para ser utilizado expresamente para las aulas (Salinas, 1992) sino que estaban destinados a una audiencia amplia, catalogados como de "divulgación cultural" en la división de Cebrián (1987; citado por Bravo, 1996) y que, por lo tanto, no seguían ninguna programación curricular de la enseñanza reglada. Por otro lado, aunque no puedan considerarse propiamente películas, muchos se acercan al documental con esquemas muy cinematográficos.

Buscando una mejora de los futuros productos audiovisuales a diseñar, la presente investigación evaluativa tuvo por objetivo principal identificar los recursos que ayudan en mayor o menor medida a los estudiantes a entender los contenidos. De este modo, sería posible poder aplicar las conclusiones obtenidas en futuros audiovisuales similares concebidos ahora sí como vídeos didácticos, con una intencionalidad netamente didáctica.

\section{Metodología}

Se realizó una investigación evaluativa de corte mixto con el fin de planificar, mejorar y justificar nuevos productos (McMillan \& Schumacher, 2005) similares a la Fundación Integra.

\subsection{Participantes}

La población fue estudiantes de $4 .^{\circ}$ Educación Secundaria Obligatoria (ESO) de la Región de Murcia, que se eligió porque en este nivel educativo se imparte la Edad Contemporánea y los estudiantes ya poseen conocimientos básicos relacionados con la geología (impartidos en $3 .^{\circ} \mathrm{ESO}$ en Biología y Geología) (LOMCE), siendo estos los temas centrales de los audiovisuales seleccionados. La muestra participante fue 120 estudiantes (hombres: $n=52,44,1 \%$; mujeres: $n=66,55,9 \% ; 2$ casos perdidos) distribuidos en cinco grupos provenientes de tres centros educativos seleccionados por conveniencia (un centro público ubicado en zona urbana, un centro público en zona rural, y un centro privado concertado). Puesto que no todos los estudiantes acudieron al visionado de todos los audiovisuales, se registró una muestra final en cada uno (Tabla 1). La edad media fue 15,7 años, la moda de la edad fue 15 (55\% de los casos), y el rango de edad fue 3 (entre 15 y 18 años). El contexto socioeconómico oscilaba entre medio-bajo y medio. Durante el visionado y recogida de información, los estudiantes se mostraron atentos y disciplinados, y no hubo incidencias que desvirtuaran los resultados finales. 
Tabla 1

Estudiantes que participaron en el visionado de cada audiovisual según centro educativo

\begin{tabular}{|c|c|c|c|c|c|}
\hline \multirow[t]{2}{*}{ Centro } & \multicolumn{2}{|c|}{ Audiovisual 1 (AV1) } & \multicolumn{2}{|c|}{ Audiovisual 2 (AV2) } & \multirow{2}{*}{$\begin{array}{c}\begin{array}{c}\text { Audiovisual } 3 \\
\text { (AV3) }\end{array} \\
\mathrm{n}\end{array}$} \\
\hline & $\mathrm{n}$ & $\%$ & $\mathrm{n}$ & $\%$ & \\
\hline Centro_1 & 49 & 40,8 & 51 & 43,6 & 40 \\
\hline Centro_2 & 34 & 28,3 & 32 & 27,4 & 34 \\
\hline Centro_3 & 37 & 30,8 & 34 & 29,1 & 32 \\
\hline TOTAL & 120 & & 117 & & 106 \\
\hline
\end{tabular}

\subsection{Instrumentos}

En este apartado se presentan los audiovisuales empleados, el instrumento de recogida de información, y el procedimiento.

Se seleccionaron tres documentales (AV1: «Seda y Esparto en la Región de Murcia», AV2: «Casas-Torre y Ermitas de la Región de Murcia», y AV3: «Murcia, enclave geológico») por estar relacionados con contenidos de Geografía e Historia y tener estilos narrativos distintos. Así, AV1 tiene formato videoblog con enfoque juvenil y reportero «youtuber» que actúa de entrevistador a un experto; AV2 tiene planteamiento tradicional con voz en off y dos presentadores que desgranan el contenido mostrado en imágenes; y AV3 tiene carácter cinematográfico. Para evitar pérdida de atención del alumnado por un visionado prolongado y poder comparar los audiovisuales entre sí, se seleccionaron intencionalmente secuencias de cada audiovisual que mostraran contenidos significativos, de fácil asimilación, y en los que se empleara un mismo tipo de recurso. Las secuencias seleccionadas no superaron los 15 minutos para cada audiovisual (AV1: 37'-52'; AV2: 1'-17'; AV3: 1'$\left.15^{\prime}\right)$.

Para el proceso de recogida de información se diseñó un instrumento ad hoc compuesto por: (i) datos identificativos del participante; (ii) entre 16 y 19 preguntas abiertas para medir la comprensión de aspectos temporales/históricos o espaciales/geográficos, de aspectos conceptuales asociados al uso de estrategias y recursos audiovisuales (vOz en off, presentador/a a cámara, recreación, fotografía/imagen, infografía, texto adicional), y de contenidos específicos clave de la secuencia visualizada; y (iii) preguntas de opinión (diferencial semántico y pregunta abierta) sobre diversos aspectos de los audiovisuales y recursos empleados. Dado el objetivo de esta investigación, no se analizaron las preguntas de opinión (punto iii).

En la elaboración del instrumento los investigadores actuaron como expertos hasta alcanzar un consenso en cuanto a la adecuación, claridad y relevancia de las preguntas puesto que poseían experiencia en la enseñanza de historia y geografía en Educación Secundaria. Posteriormente, otro experto ajeno a la investigación y experto en historia y geografía en Educación Secundaria realizó una segunda validación del instrumento. Finalmente, este se envió a la Fundación Integra para una revisión final, la cual sugirió ajustes menores. El instrumento se adaptó a las peculiaridades de cada audiovisual, si bien presentó la misma estructura en los tres casos (http://www.regmurcia.com/docs/CuestionariosAAVV.pdf).

En relación al procedimiento seguido, se contactó con los centros para explicarles el objetivo y características de la investigación y obtener su consentimiento para participar. Además, se obtuvo consentimiento informado de los padres de los participantes. A continuación, se procedió al concierto de visitas para el visionado de los audiovisuales y recogida de datos. Cada grupo fue visitado en tres ocasiones (una por audiovisual) por dos investigadores formados en la correcta muestra de los audiovisuales y administración del instrumento. A fin de distanciar la exposición a los materiales de los participantes, se planificaron visitas cada dos semanas. Se requirió un mes y medio para recoger datos. Se alternó el orden de visionado de los audiovisuales en los cinco grupos para minimizar sesgos. 


\subsection{Método}

En relación al análisis de los datos obtenidos, las cuestiones sobre conocimientos específicos se analizaron cualitativamente para concluir si eran correctas/parcialmente correctas/incorrectas. Los investigadores examinaron independientemente varios cuestionarios de los tres audiovisuales para poder emitir un juicio independiente sobre el nivel de corrección de las respuestas y así alcanzar un acuerdo sobre cómo valorar las mismas. Esto permitió establecer una rúbrica de respuestas que permitiera una corrección homogénea. Se tuvieron en cuenta las respuestas en blanco para llevar registro de preguntas que no habían sido respondidas con éxito. Se asumió que la mayoría no fueron contestadas por desconocimiento de la respuesta; de hecho, muchos participantes explicitaron que no recordaban la respuesta o no la sabían. El análisis en función del nivel de corrección de las respuestas permitió sistematizar la corrección y obtener una puntuación final numérica de los conocimientos de los estudiantes sobre cada audiovisual.

\section{Resultados}

Los primeros resultados muestran el nivel de corrección de las respuestas de los participantes por audiovisual (Tabla 2). El AV3 obtuvo el mayor porcentaje de preguntas sin respuesta y el menor porcentaje de respuestas correctas o parcialmente correctas (23,3\% agrupado), mientras que el AV1 tuvo el mayor porcentaje de respuestas correctas y parcialmente correctas (59,4\% agrupado) y menor porcentaje de respuestas incorrectas o sin respuesta.

Tabla 2

Nivel de corrección de respuestas por audiovisual

\begin{tabular}{lccc}
\hline Audiovisual & \multicolumn{2}{c}{ Respuestas } & \\
\cline { 2 - 3 } & Correctas & Parcialmente correctas & Incorrectas \\
\hline AV1 & $48,0 \%$ & $11,4 \%$ & $15,6 \%$ \\
\hline AV2 & $31,1 \%$ & $14,2 \%$ & $18,5 \%$ \\
\hline AV3 & $16,0 \%$ & $7,3 \%$ & $18,0 \%$ \\
\hline
\end{tabular}

Con el fin de poder analizar el grado de dificultad de cada audiovisual y si existe relación entre la estrategia utilizada para presentar contenidos y el grado de corrección de las respuestas, se procedió a analizar cada audiovisual pormenorizadamente (Tabla 3, 4 y 5). Los resultados muestran las preguntas agrupadas según el tipo de contenido y la estrategia empleada en cada audiovisual, así como la suma del porcentaje de respuestas correctas y parcialmente correctas asociadas a cada ítem.

En cuanto al AV1 (Tabla 3), se observaron ciertas tendencias iniciales pues las preguntas asociadas directamente al hilo argumental de la historia $(3.13,3.14,3.18)$ obtuvieron un alto porcentaje de respuesta correcta y parcialmente correcta, lo que sugiere que la historia es entendida y seguida por la mayoría de participantes. Sin embargo, las cuestiones asociadas a la época y momento en el que suceden diversas acciones y hechos $(3.4,3.5)$ tienen un bajo porcentaje de corrección teniendo en cuenta la tendencia propia del audiovisual. Así, sólo algunos participantes recordaron el momento aproximado en el que se desarrollaba la acción (3.4) o la década en la que se inició la crisis del esparto (3.5). Esta información fue suministrada por el narrador sin ningún soporte visual o textual. Sin embargo, preguntados por quiénes denominaron a la comarca de Cartagena como «Campus Spartarius» (3.15), 65\% de los participantes contestó correctamente, siendo la información presentada por el narrador (voz en off) apoyado por imágenes asociadas a la civilización romana que aparecían en pantalla.

Pese a que los participantes no recordaron en demasía cuándo se produjo la crisis del esparto (3.5), una mayoría sí recordó las causas (3.6), que fueron explicadas por el narrador y a la vez presentadas mediante el uso de la prensa del momento (donde eran resaltados los titulares) e imágenes. 
En cuanto a las cuestiones geográficas, las preguntas 3.7 y 3.8 obtuvieron unos elevados porcentajes de corrección. La información referida a estas cuestiones aparecía impresa en pantalla mediante cartografía y narrada simultáneamente. La pregunta 3.7 incluía además información sobreimpresa con el nombre de las regiones. Son llamativas las divergencias en los porcentajes de respuesta al cruzar la respuesta 3.7 con la 3.16. Concretamente, el porcentaje de respuestas correctas en el segundo se reduce pese a compartir respuesta, por lo que parece que el hecho de que la fecha fuera mencionada únicamente por el narrador produjo mayor dificultad. La cuestión relacionada con aspectos geográficos referida al municipio principal en la transformación del esparto (3.17) fue únicamente narrada por la vOz en off en los últimos minutos del metraje y evocada correctamente por la mitad de los estudiantes.

Existen preguntas con alto porcentaje de acierto $(3.3,3.15)$, cuya información fue únicamente narrada por voz en off y tuvo escaso acompañamiento visual, pero que cuya temática parece interesar al alumnado (enfermedades, productos que reconoce en la actualidad). No sucede así con aspectos más técnicos (profesiones, herramientas específicas), que el alumnado no recuerda con facilidad (3.9, 3.11).

Por último, cabe señalar la pauta de respuesta del ítem 3.10 (64,1\%), que muestra que solo $23,2 \%$ de los participantes pudo indicar el nombre de la labor desarrollada por la protagonista en la fabricación del esparto (respuesta correcta), mientras que 40,8\% pudo realizar una descripción minuciosa de su trabajo (respuesta parcialmente correcta) acorde a la recreación transmitida en AV1.

Tabla 3

Preguntas según contenido, estrategia utilizada, y porcentaje de respuestas correctas y parcialmente correctas sobre AV1

\begin{tabular}{|c|c|c|c|c|c|c|c|}
\hline Pregunta & & & & Estrat & & & \\
\hline $\begin{array}{l}\text { Tipo de } \\
\text { contenido }\end{array}$ & Ítem & $\begin{array}{l}\text { Voz } \\
\text { en } \\
\text { Off }\end{array}$ & $\begin{array}{l}\text { Presentador a } \\
\text { cámara }\end{array}$ & Recreación & Imagen & $\begin{array}{l}\text { Infografía } \\
\text { (sin texto) }\end{array}$ & Texto \\
\hline
\end{tabular}

\begin{tabular}{|c|c|c|c|c|c|c|}
\hline \multirow[t]{3}{*}{ Hilo argumental } & 3.13 & & $\mathrm{x}$ & & & \\
\hline & 3.14 & $\mathrm{x}$ & & & & $\mathrm{x}$ \\
\hline & 3.18 & $\mathrm{x}$ & & & & \\
\hline \multirow{3}{*}{$\begin{array}{l}\text { Aspectos } \\
\text { cronológicos }\end{array}$} & 3.4 & $\mathrm{x}$ & & & & \\
\hline & 3.5 & $\mathrm{x}$ & & & & \\
\hline & 3.15 & $\mathrm{x}$ & & $\mathrm{x}$ & & \\
\hline \multirow{4}{*}{$\begin{array}{l}\text { Aspectos } \\
\text { geográficos }\end{array}$} & 3.7 & $\mathrm{x}$ & & & $\mathrm{x}$ & $\mathrm{x}$ \\
\hline & 3.8 & $\mathrm{x}$ & & & $\mathrm{x}$ & \\
\hline & 3.16 & $\mathrm{x}$ & & & $\mathrm{x}$ & \\
\hline & 3.17 & $\mathrm{x}$ & & & & \\
\hline \multirow{6}{*}{$\begin{array}{l}\text { Contenidos } \\
\text { específicos }\end{array}$} & 3.3 & $\mathrm{x}$ & & & & \\
\hline & 3.6 & $\mathrm{x}$ & & $\mathrm{x}$ & & $\mathrm{x}$ \\
\hline & 3.9 & $\mathrm{x}$ & $\mathrm{x}$ & & & \\
\hline & 3.10 & $\mathrm{x}$ & $\mathrm{x}$ & & & \\
\hline & 3.11 & $\mathrm{x}$ & $\mathrm{x}$ & & & \\
\hline & 3.12 & $\mathrm{x}$ & $\mathrm{x}$ & & & \\
\hline
\end{tabular}

Fuente: Elaboración propia. 
En relación con el AV2 (Tabla 4), es difícil obtener un patrón de respuesta claro al comparar el porcentaje de respuesta obtenido con las estrategias utilizadas. Las respuestas con un alto porcentaje de corrección aparecen asociadas, con la misma frecuencia, tanto a aquella información dada por el presentador mirando a cámara como a aquella reforzada con infografías, imágenes y texto. Así, llama la atención que $28,2 \%$ de los participantes recordara el momento en el que se debía realizar el pago del rento (3.18), dato que aparece en el último minuto del metraje dado por el presentador mirando a cámara entre dos explicaciones de cierta extensión narradas con voz en off.

El único aspecto que parece mostrar cierta homogeneidad es el referido a la introducción de cartografía, con resultados positivos $(3.8,3.13)$. La comprensión temporal sigue siendo baja $(3.7$, 3.18), pese a que fuera reforzada con una infografía a modo de cartografía donde aparecían representadas simbólicamente las culturas presentes en la Península Ibérica en el momento en cuestión.

Tabla 4

Preguntas según contenido, estrategia utilizada, y porcentaje de respuestas correctas y parcialmente correctas sobre AV2

\begin{tabular}{|c|c|c|c|c|c|c|c|}
\hline \multicolumn{2}{|l|}{ Pregunta } & \multicolumn{6}{|c|}{ Estrategia } \\
\hline $\begin{array}{l}\text { Tipo de } \\
\text { contenido }\end{array}$ & Ítem & $\begin{array}{c}\text { Voz } \\
\text { en } \\
\text { Off }\end{array}$ & $\begin{array}{l}\text { Presentador a } \\
\text { cámara }\end{array}$ & Recreación & Imagen & $\begin{array}{l}\text { Infografía } \\
\text { (sin texto) }\end{array}$ & Texto \\
\hline Hilo argumental & 3.10 & & $\mathrm{x}$ & & & & \\
\hline \multirow{2}{*}{$\begin{array}{l}\text { Aspectos } \\
\text { cronológicos }\end{array}$} & 3.7 & $\mathrm{x}$ & & & & $\mathrm{x}$ & \\
\hline & 3.18 & & $\mathrm{x}$ & & & & \\
\hline \multirow{2}{*}{$\begin{array}{l}\text { Aspectos } \\
\text { geográficos }\end{array}$} & 3.8 & $\mathrm{x}$ & & & & $\mathrm{x}$ & $\mathrm{x}$ \\
\hline & 3.13 & $\mathrm{x}$ & & & & $\mathrm{x}$ & $\mathrm{x}$ \\
\hline \multirow{11}{*}{$\begin{array}{l}\text { Contenidos } \\
\text { específicos }\end{array}$} & 3.5 & $*$ & & & & & \\
\hline & 3.6 & $\mathrm{x}$ & & & $\mathrm{x}$ & & $\mathrm{x}$ \\
\hline & 3.9 & $\mathrm{x}$ & & & & & $\mathrm{x}$ \\
\hline & 3.11 & & $\mathrm{x}$ & & & & \\
\hline & 3.12 & & $\mathrm{x}$ & & & & \\
\hline & 3.14 & $\mathrm{x}$ & & & $\mathrm{x}$ & $\mathrm{x}$ & $\mathrm{x}$ \\
\hline & 3.15 & $\mathrm{x}$ & & $\mathrm{x}$ & & & \\
\hline & 3.16 & $\mathrm{x}$ & & $\mathrm{x}$ & $\mathrm{x}$ & & \\
\hline & 3.17 & & $\mathrm{x}$ & & & & \\
\hline & 3.18 & & $\mathrm{x}$ & & & & \\
\hline & 3.19 & $\mathrm{x}$ & & & & & $\mathrm{x}$ \\
\hline
\end{tabular}

Fuente: Elaboración propia.

En el caso del AV3 (Tabla 5), aproximadamente uno de cada cuatro participantes recordó el hecho anecdótico de que el presentador se encontrara sobre una canoa remando en el río al principio del vídeo (3.9). Sin embargo, fueron pocos más los capaces de indicar la cordillera de la que forma parte la Región de Murcia (3.6), la Cordillera Bética, dato repetido en múltiples ocasiones con diversos soportes (texto, voz en off, presentadores, mapa) y protagonista indiscutible del audiovisual. 
En relación con las preguntas sobre cronología (3.3, 3.7, 3.8, 3.10), los porcentajes de respuesta pueden ser considerados positivos teniendo en cuenta la propia tendencia de este documental (cf. Tabla 2) y los resultados obtenidos en AV1 y AV2. En concreto, el 22,6\% de los participantes reprodujo correctamente la edad de la tierra (3.7) y el 28,3\% señaló con precisión el dato sobre la aparición del Homo Sapiens (3.10). Igualmente, el concepto "precámbrico" (3.3) no aparece narrado ni explicado en ningún momento (solo impreso sobre la línea del tiempo) y sin embargo es recordado como era geológica por $16 \%$ de los participantes. Toda esta información aparece al principio y al final de una secuencia donde una línea del tiempo es construida progresivamente para explicar las eras geológicas. En esta secuencia se menciona también la era de los grandes reptiles (3.8). Dicha información aparece 43 segundos después del inicio de dicha secuencia. Solo 4,7\% de los participantes recordó la era secundaria o mesozoico (respuesta correcta), y 11,3\% aludió al periodo Jurásico en su lugar (respuesta parcialmente correcta). La cantidad de información aportada a lo largo de este recurso durante un espacio prolongado de tiempo (la secuencia completa de construcción de la línea del tiempo dura 1 minuto) hace que la información intermedia parezca desvanecerse entre los innumerables datos aportados.

En relación con los contenidos geográficos, se destaca el comparativamente elevado porcentaje de corrección en las respuestas asociadas a la parte del Península Ibérica sumergida (3.4). Con mayor o menor precisión, $37,8 \%$ de los participantes recordó la respuesta, que curiosamente no había sido narrada sino representada en dos infografías a lo largo del documental. Mayor dificultad tuvieron al identificar la línea de costa en el Sureste durante el Triásico (3.5) pese a que esto fue narrado por los presentadores en dos ocasiones. Esta información no aparecía vinculada a ningún recurso gráfico. Por el contrario, y pese a compartir respuesta, uno de cada cuatro participantes recordó la zona emergida donde se pasearon los grandes reptiles (3.12). El hecho de que la presentadora mencionara que los dinosaurios se pasearon por localidades de la Región de Murcia pudo despertar la atención sobre esta información concreta.

Por último, es llamativo el comparativamente elevado porcentaje de respuestas correctas obtenidas en relación con la formación de la Cordillera Bética (3.13). Parte de los participantes fue capaz de describir el proceso de formación (respuesta parcialmente correcta) y de incorporar algunos de los nombres de las placas que intervinieron en el proceso (respuesta correcta). Parece que las infografías pudieron ser de ayuda para entender los procesos más complejos. Sin embargo, este recurso no siempre parece funcionar de la misma manera. Así lo muestra el ítem 3.14, cuya información se muestra en un mapa donde la información textual aparece a modo de leyenda y no sobreimpresa en el territorio. Esto quizás pudo dificultar el recuerdo visual de la información.

Tabla 5

Preguntas según contenido, estrategia utilizada, y porcentaje de respuestas correctas y parcialmente correctas sobre AV3

\begin{tabular}{lccccccc}
\hline Pregunta & \multicolumn{7}{c}{ Estrategia } \\
\hline $\begin{array}{l}\text { Tipo de } \\
\text { contenido }\end{array}$ & Ítem & $\begin{array}{c}\text { Voz } \\
\text { en } \\
\text { Off }\end{array}$ & $\begin{array}{c}\text { Presentador a } \\
\text { cámara }\end{array}$ & Recreación & Imagen & $\begin{array}{c}\text { Infografía } \\
\text { (sin texto) }\end{array}$ & Texto \\
\hline Hilo argumental & 3.9 & & $\mathrm{x}$ & & $\mathrm{x}$ & $\mathrm{x}$ \\
$\begin{array}{l}\text { Aspectos } \\
\text { cronológicos }\end{array}$ & 3.3 & 3.7 & $\mathrm{x}$ & $\mathrm{x}$ & $\mathrm{x}$ & $\mathrm{x}$ \\
& 3.8 & $\mathrm{x}$ & $\mathrm{x}$ & $\mathrm{x}$ \\
& 3.10 & & $\mathrm{x}$ & $\mathrm{x}$ & $\mathrm{x}$ \\
& 3.2 & $\mathrm{x}$ & & $\mathrm{x}$ &
\end{tabular}




\begin{tabular}{lccccc} 
Aspectos & 3.4 & & $\mathrm{x}$ & \\
geográficos & 3.5 & $\mathrm{x}$ & $\mathrm{x}$ & $\mathrm{x}$ \\
& 3.12 & $\mathrm{x}$ & $\mathrm{x}$ & & \\
\hline $\begin{array}{l}\text { Contenidos } \\
\text { específicos }\end{array}$ & 3.16 & & $\mathrm{x}$ & $\mathrm{x}$ & $\mathrm{x}$ \\
& 3.1 & $\mathrm{x}$ & & & \\
& 3.11 & & $\mathrm{x}$ & $\mathrm{x}$ & $\mathrm{x}$ \\
& 3.13 & $\mathrm{x}$ & & $\mathrm{x}$ & \\
\hline 3.14 & & $\mathrm{x}$ & & \\
\hline
\end{tabular}

Fuente: Elaboración propia.

\section{Discusión}

Tras el análisis realizado se pueden extraer conclusiones en relación con la incidencia de cada recurso analizado en el aprendizaje de los participantes y dar así respuesta al objetivo de esta investigación. Primero, imágenes acompañadas de voz en off fue uno de los recursos más repetidos en los tres audiovisuales. Su uso parece reforzar la comprensión y el recuerdo del contenido que ilustra. Este resultado parece coincidir en general con los de otras investigaciones que evidencian que las imágenes se recuerdan mejor que los textos (Bashman \& Treadwell, 1995, citado en Mitra et al. 2010). Como si de un libro se tratara, las imágenes sirven para aumentar el número de ideas del espectador, dando sentido muchas veces a aquello que se escucha (Eilam, 2012). Si además esas imágenes se corresponden con fotografías propias de la época sobre la que se desarrolla la acción, el efecto formativo parece mejorar.

Además de las imágenes, por su relevancia en la comprensión de la historia y la geografía, cabe destacar dos recursos específicos: los mapas y las líneas del tiempo. La abstracción necesaria para comprender fenómenos tan alejados en el tiempo requiere información gráfica de apoyo (Abelman, 1990). Así, por ejemplo, los aspectos temporales o cronológicos del AV1 y AV2 son escasamente recordados, porcentaje que mejora en el AV3. Estos datos coinciden con buena parte de las investigaciones empíricas recientes sobre la utilidad de este recurso, en las que su uso en las actividades de Educación Primaria o Secundaria mejora la comprensión temporal (GrootReuvekamp, Ros \& Boxtel, 2017; Groot-Reuvekamp, Ros, Boxtel \& Oort, 2017; Hoodless, 1996). A pesar de que se reduzca la comprensión del concepto "tiempo" a un modelo lineal y encorsetado por los cajones artificiales que son las épocas, la línea del tiempo parece facilitar la contextualización temporal. Sin embargo, los datos obtenidos en AV3 también parecen mostrar el peligro de incorporar excesivos datos a colación de este recurso. Este dato está relacionado con el denominado «redundancy effect», por el que el aprendizaje parece verse obstaculizado conforme los estudiantes tienen estímulos redundantes (Jin, 2012; Lang, 1995; Mayer, Heiser \& Lonn, 2001).

Respecto a la cartografía, las respuestas sobre información de los mapas obtuvieron unos porcentajes de acierto bastante elevados. El único matiz que parece desgajarse de este resultado está relacionado con el modo de presentar la información en los mismos. La información debe estar preferiblemente integrada en el mapa y no a modo de leyenda (como así parecen mostrar los datos en el AV3), pues resultan ineficaces para el recuerdo de la información si los datos que ofrecen no aportan claridad. En la enseñanza de la Historia, y especialmente de la Geografía, los mapas son fundamentales, y así se consideran desde hace décadas (Fairgrieve, 1932), pero para ello estos han de contener información muy precisa, visual, limpia, despertar interés y además ofrecer explicaciones (Sancho, 1996). A pesar de que a priori se pueda pensar que a las edades de los participantes los mapas 
son superfluos, recientes investigaciones (Dijk, Schee, Trimp \& Zijpp, 1994; Gökçe, 2016; Piñeiro \& Melón, 2002) aportan datos clarificadores sobre las serias dificultades que los estudiantes tienen a la hora de trabajar con mapas o ubicar determinados elementos geográficos en el espacio.

Por su parte, las infografías han demostrado su utilidad pues se puede aplicar la misma reflexión que sobre la cartografía: un uso prolongado del mismo recurso, en el que la infografía se prolonga en el tiempo y se añade un exceso de información, parece reducir su eficacia. La concisión y sencillez se convierten en herramientas básicas para su diseño si se pretende que realmente ofrezcan algo distinto a las imágenes estáticas (Bétrancourt \& Tversky, 2000; Höffler \& Leutner 2007).

Pese a lo que en principio puede parecer, la eficacia de la información impresa en pantalla parece depender de la cantidad, momento y complejidad del contenido. Así, la información escrita resulta eficaz a la hora de recordar contenidos en el caso del AV3 y más anecdóticos en AV1 y AV2.

El último aspecto a valorar es relativo al rol y características de actores y presentadores. El uso de presentador es una fórmula que suele funcionar en los vídeos didácticos, especialmente si este interactúa con el espectador (Johnson, 1989). Estudios recientes sobre vídeos con profesor presente o no en pantalla (van Gog, Verveer \& Verveer, 2014; Wang \& Antonenko, 2017) ratifican que este recurso potencia la motivación y capacidad de atención. Otros estudios (Kemp \& Dayton, 1985) apuntan que este recurso es más eficaz si la audiencia se identifica con el narrador. Los presentadores del AV2 y AV3 se muestran más cercanos, ofrecen información puntual y en momentos muy determinados, y no sostiene narraciones prolongadas. Estos parecen captar mejor la atención del alumnado, aunque también se puede asociar al momento en el que se produce la interacción: las apelaciones directas puntuales del presentador a cámara tras una larga explicación parecen captar la atención y favorecer el recuerdo. La sustitución de presentadores por actores recreando la época parece haber ayudado también a recordar algunos contenidos presentes en los vídeos.

\section{Conclusiones}

Siguiendo la clasificación ya clásica de Bruner (1964) sobre los modos de aprendizaje, se podría prever que dadas las edades de los participantes analizados, estos serían capaces de procesar y emplear la información procedente del modo simbólico, es decir, a través del lenguaje oral o escrito. Sin embargo, en base a los resultados de esta investigación, parece que los participantes recuerdan mejor los elementos que se presentaban acompañados de soporte gráfico (mapas, fotografías, infografías, etc.), propios del modo icónico de aprendizaje. Al utilizar diferentes canales para transmitir una misma información, los estímulos se incrementan y se produce una mejor retención (Seel, 2008). Evaluaciones similares de videojuegos educativos también apuestan por esta necesidad de mostrar la información por varias vías (Egea, Arias, \& García, 2017). Por ese motivo, futuras producciones audiovisuales podrían tener en cuenta este tipo de anclajes visuales que, combinados con explicaciones, ayudan en los procesos cognitivos de los espectadores (Mayer, 1997). Estas explicaciones deben ser claras y concisas ya que presentar mucha cantidad de información en poco tiempo parece ser contraproducente para el aprendizaje al provocar el mencionado «redundancy effectr. La falta de relevancia de cierta información, aunque esta pueda llegar a través de varios estímulos, provoca distracción (Seel, 2008) y, por tanto, peor aprendizaje. Es lo que Mayer (2009) denominó «coherence principle». Además, Laurillard (2001) señala la importancia de la sinergia entre imagen y argumento, que solo llega a funcionar cuando la imagen dura el tiempo que permite asimilar la información o el concepto descrito.

Estos mismos principios parecen ser aplicables a todos los recursos analizados, ya sean imágenes, recreaciones, actores o presentadores. El uso de diferentes recursos, la concreción de la información, y la cercanía del lenguaje parecen redundar en una mejor recepción del mensaje.

No obstante, este campo de investigación desde un punto de vista evaluativo está aún por construir, aún hay mucho que decir. A pesar de que son muy habituales las investigaciones sobre los beneficios de la innovación en didáctica de las ciencias sociales (y en todas las disciplinas), aún es necesario un mayor recorrido que analice la verdadera "efectividad que trae consigo en la práctica" cada innovación (Luna, en prensa). 
Entre las limitaciones cabe asumir las dificultades organizativas y de financiación que estudios tan ambiciosos como este conllevan. Por último, entre los retos y oportunidades que se abren en el campo cabe destacar la conveniencia de llevar a cabo estudios con muestras de mayor tamaño seleccionadas aleatoriamente, trabajos monográficos centrados en analizar un determinado recurso, de cara a ratificar o no estos resultados o comparar los mismos con estudios similares que incluyan videojuegos y comprobar si existen diferencias entre ambos medios pues el lenguaje audiovisual actualmente va más allá del cine y el documental.

\section{Referencias bibliográficas}

Abelman, R. (1990). You can't get there from here: Children's understanding of time-leaps on television. Journal of Broadcasting \& Electronic Media, 34(4), 469-476. 10.1080/08838159009386755

Aitken, S. C. (1994). I'd rather watch the movie than read the book. Journal of Geography in Higher Education, 18(3), 291-307. 10.1080/03098269408709269

Algeo, K. (2007). Teaching Cultural Geography with Bend It Like Beckham. Journal of Geography, 106(3), 133-143. 10.1080/00221340701632781

Anderson, D. (1998). Educational television is not an oxymoron. Annals of the American Academy of Political and Social Science, 557, 24-38.

Ball, S., \& Bogatz, G. A. (1970). The first year of Sesame Street: an evaluation. Princeton, N.J.: Educational Testing Service.

Ballesteros, C. (2016). Los medios audiovisuales: funciones didácticas y principios metodológicos para su integración en los procesos de enseñanza y aprendizaje. IJERI: International Journal of Educational Research and Innovation, 6, 58-70.

Bashman, J. G., \& Treadwell, T. W. (1995). Assessing the effectiveness of a psychodrama training video. Journal of Group Psychotherapy, Psychodrama and Sociometry, 48, 61-68.

Bétrancourt, M., \& Tversky, B. (2000). Effect of computer animation on users' performance: a review. Le Travail Humain, 63(4), 311-329.

Bravo, L. (1996). ¿Qué es el vídeo educativo? Comunicar: Revista científica iberoamericana de comunicación y educación, 6, 100-105.

Breu, R. (2012). La historia a través del cine. 10 propuestas didácticas para secundaria y bachillerato. Barcelona: Ed. Graó.

Bruner, J. S. (1964). The course of cognitive growth. American Psychologist, 19(1), 1-15.

Carrasco, E. (2017). Pedagogical proposal to support the teaching of history with audiovisual content including video game advertising: the example of Assassin`s Creed IV: Black flag and privateer Amaro Pargo. En A. V. Stavros (Ed.), Advances in Communications and Media Research (pp. 145-164). Hauppauge, NY: Nova Science Publishers.

Castro, M. (2006). El cine como instrumento de socialización. A Parte Rei, 47, 1-6.

Cole, C. F., \& Lee, J. H. (Eds.). (2016). The Sesame effect: the global impact of the longest street in the world. New York, NY: Routledge.

Connell, D. D., \& Palmer, E. L. (1971). Sesame Street. A case study. En J. D. Halloran \& M. Gurevitch (Eds.), Broadcaster / researcher co-operation in mass communication research: a report on an international seminar held at the University of Leicester, England December 17th - 21st, 1970 (pp. 66-85). Leicester: Centre for Mass Communication Research, University of Leicester.

Del Alcàzar, J. (2012). Historia desde el cine [y con la literatura] para la educación. Revista Brasileira de Estudos Pedagógicos, 93(235), 645-666. 10.1590/S2176-66812012000400007.

Dijk, H. van, Schee, J. van der, Trimp, H., \& Zijpp, T. van der. (1994). Map skills and geographical knowledge. International Research in Geographical and Environmental Education, 3(1), 68-80. 10.1080/10382046.1994.9964928

Donnelly, D. (2014). Using Feature Film in the Teaching of History: The Practitioner DecisionMaking Dynamic. Journal of International Social Studies, 4(1), 17-27. 
Durbin, C. (1995). Using televisual resources in geography. Teaching Geography, 20(3), 118-121.

Egea, A., Arias, L., \& García, A. J. (2017). Videojuegos, historia y patrimonio: primeros resultados de una investigación educativa evaluativa en educación secundaria. Revista Interuniversitaria de Investigación en Tecnología Educativa, 2, 28-40. 10.6018/riite/2017/283801.

Egea, A., Pernas, S., \& Arias, L. (2013). La aplicación del cine en las aulas: la vida de los monasterios medievales a través de "El nombre de la Rosa". En J. J. Díaz, A. Santisteban, \& A. Cascarejo, Medios de Comunicación Y Pensamiento Crítico. Nuevas Formas de Interacción Social (pp. 539-549). Alcalá de Henares: Universidad de Alcalá.

Eilam, B. (2012). Teaching, learning, and visual literacy: the dual role of visual representation. New York, NY: Cambridge University Press.

Fairgrieve, J. (1932). The use of films in teaching. Geography, 17(2), 129-140.

Fairgrieve, J. (1942). The Supply of Films for Teaching Regional Geography. Sight and Sound, 10(40), 81-84.

Fisch, S. M. (2004). Children's learning from educational television: Sesame Street and beyond. Mahwah, NJ: L. Erlbaum Associates.

Gambari, A. I., Shittu, A. T., Daramola, F. O., \& James, M. (2016). Effects of Video-Based Cooperative, Competitive and Individualized Instructional Strategies on the Performance of Senior Secondary Schools Students in Geometry. Malaysian Online Journal of Educational Sciences, 4(4), 31-47.

Gambari, A. I., Yaki, A. A., Gana, E. S., \& Ughovwa, Q. E. (2014). Improving Secondary School Students' Achievement and Retention in Biology through Video-Based Multimedia Instruction. InSight: A Journal of Scholarly Teaching, 9, 78-91.

Gökçe, N. (2016). Social Studies in Improving Students' Map Skills: Teachers' Opinions. Educational Sciences: Theory and Practice, 15(5), 1345-1362. doi: 10.12738/estp.2015.5.0071

Groot-Reuvekamp, M. de, Ros, A., \& Boxtel, C. van. (2017). Improving Elementary School Students' Understanding of Historical Time: Effects of Teaching With "Timewise." Theory \& Research in Social Education, 45(4), 1-33. doi: 10.1080/00933104.2017.1357058

Groot-Reuvekamp, M. de, Ros, A., Boxtel, C. van, \& Oort, F. (2017). Primary school pupils' performances in understanding historical time. Education 3-13, 45(2), 227-242. doi: 10.1080/03004279.2015.1075053

Grupo «Imágenes de la Historia», Gorgues, R. \& Goberna, J.J. (1998). El cine en la clase de historia. Comunicar: Revista científica iberoamericana de comunicación y educación, 11, 87-93.

Höffler, T. N., \& Leutner, D. (2007). Instructional animation versus static pictures: A meta-analysis. Learning and Instruction, 17(6), 722-738. doi: 10.1016/j.learninstruc.2007.09.013

Hollingham, S. (1997). Using feature films in geography teaching. Teaching Geography, 22(3), 131-133. Hoodless, P. (1996). Time and timelines in the primary school. London: Historical Association.

Ijaz, K., Bogdanovych, A., \& Trescak, T. (2017). Virtual worlds vs books and videos in history education. Interactive Learning Environments, 25(7), 904-929. doi: 10.1080/10494820.2016.1225099

Jin, P. (2012). Redundancy Effect. In N. M. Seel (Ed.), Encyclopedia of the Sciences of Learning (pp. 27872788). Boston, MA: Springer US. doi: 10.1007/978-1-4419-1428-6_200

Johnson, D. A. (1989). Training by Television. Training and Development Journal, 43(8), 64-68.

Kemp, J. E., \& Dayton, D. K. (1985). Planning and producing instructional media (5th ed). New York, NY: Harper \& Row.

Lang, A. (1995). Defining Audio/Video Redundancy From a Limited- Capacity Information Processing Perspective. Communication Research, 22(1), 86-115. 10.1177/009365095022001004.

Laurillard, D. (2013). Rethinking university teaching: a conversational framework for the effective use of learning technologies. Hoboken: Taylor and Francis.

López, M., \& Arias, L. (2013). Los medios audiovisuales para la enseñanza de la historia del mundo contemporáneo: la Guerra Fría. En J. J. Díaz, A. Santisteban, \& A. Cascarejo, Medios de Comunicación Y Pensamiento Crítico. Nuevas Formas de Interacción Social (pp. 479-490). Alcalá de Henares: Universidad de Alcalá. 
Luna, D. (en prensa). Innovación didáctica en Historia: un estado de la cuestión en torno a cuatro ejes temáticos. Panta Rei: revista de ciencia y didáctica de la bistoria.

Martínez, F. (2013). La historia y el cine: ¿unas amistades peligrosas? Vinculos de Historia, 2, 351-372.

Mayer, R. E. (1997). Multimedia learning: Are we asking the right questions? Educational Psychologist, 32(1), 1-19.10.1207/s15326985ep3201_1.

Mayer, R. E. (2009). Multimedia learning (2nd ed). New York, NY: Cambridge University Press.

Mayer, R. E., Heiser, J., \& Lonn, S. (2001). Cognitive Constraints on Multimedia Learning: When Presenting More Material Results in Less Understanding. Journal of Educational Psychology, 93(1), 187-198. 10.1037/0022-0663.93.1.187

McMillan, J. H., \& Schumacher, S. (2005). Investigación educativa: una introducción conceptual. Madrid: Pearson.

Mitra, B., Lewin-Jones, J., Barrett, H., \& Williamson, S. (2010). The use of video to enable deep learning. Research in Post-Compulsory Education, 15(4), 405-414. 10.1080/13596748.2010.526802.

Moya, T. (2017). Iniciativas de alfabetización cinematográfica: Una cartografía metodológica actual de las entidades dedicadas a la film literacy con públicos no profesionales en España. Revista Fuentes, 19(2), 125-138. http://dx.doi.org/10.12795/revistafuentes. 2017.19.2.09

Palma, M. T. di. (2009). Teaching Geography Using Films: A Proposal. Journal of Geography, 108(2), 47-56. 10.1080/00221340902967325.

Piñeiro, M. del R., \& Melón, M. C. R. (2002). El problema del razonamiento espacial a través del mapa. Didáctica geográfica, 5, 103-117.

Salinas, J. (1992). Diseño, producción y evaluación de vídeos didácticos. Palma: Universitat de les Illes Balears.

Sancho, J. (1996). El mapa como recurso didáctico de gran valor en la enseñanza de Geografía. Didáctica geográfica, 1, 15-20.

Seel, N. M. (2008). Empirical perspectives on memory and motivation. En J. M. Spector, M. D. Merrill, J. Van Merriënboer, \& M. P. Driscoll (Eds.), Handbook of research on educational communications and technology (3rd ed, pp. 39-54). New York, NY: Lawrence Erlbaum Associates.

Torre, S. de la (2005). Aprendizaje integrado y cine formativo. En S. de la Torre, M. A. Pujol \& N. Rajadell (coords.), El cine, un entorno educativo. Diez años de experiencias a través del cine (pp. 13-36). Madrid: Narcea.

Valero, M. del P., \& Vera, Á. L. (2005). La televisión como recurso curricular y medio de conocimiento en el área de Historia. Comunicar: Revista Cientifica de Comunicación y Educación, 13(25). 10.3916/C25-2005-204.

Valle, E. (2007). Cine e historia: sobre la utilización de los DSV en la enseñanza de la disciplina histórica. En Actas del I Congreso Internacional de Lengua, Literatura y Cultura Española: la didáctica de la enseñanza para extranjeros (pp. 445-458). Onda: JMC.

Van Gog, T., Verveer, I., \& Verveer, L. (2014). Learning from video modeling examples: Effects of seeing the human model's face. Computers \& Education, 72(Supplement C), 323-327. 10.1016/i.compedu.2013.12.004

Wang, J., \& Antonenko, P. D. (2017). Instructor presence in instructional video: Effects on visual attention, recall, and perceived learning. Computers in Human Behavior, 71, 79-89. 10.1016/j.chb.2017.01.049

Zaplana, A. (2003). Educar para la paz a través del cine de guerra. Pedagogía social: revista interuniversitaria, 10, 299-319.

Audiovisuales analizados

Alpáñez, A. (Director) (2017). Casas-Torre y Ermitas de la Región de Murcia [Documental]. España: Imagiavideo.

Bravo, A. (Director) (2017). Seda y esparto en la Región de Murcia [Documental]. España: Bravoestudios.

Muñoz, N. (Director) (2017). Murcia, enclave geológico. España: Nexus Creatividad Visual. 
38 Laura Arias-Ferrer, Alejandro Egea-Vivancos \& Fuensanta Monroy-Hernández

\section{Para citar este artículo}

Arias-Ferrer, L., Egea-Vivancos, A., \& Monroy-Hernández, F.. (2019). Evaluación de recursos audiovisuales para la enseñanza de las ciencias sociales en Educación Secundaria. Revista Fuentes, 21(1), 25-38. [Fecha de consulta: dd/mm/aa]. doi: 10.12795/revistafuentes.2019.v21.11.02 